\title{
Omega-Regular Half-positional Winning Conditions
}

\author{
Eryk Kopczyński* \\ Institute of Informatics, Warsaw University \\ erykk@mimuw . edu.pl
}

\begin{abstract}
We study infinite games where one of the players always has a positional (memory-less) winning strategy, while the other player may use a history-dependent strategy. We investigate winning conditions which guarantee such a property for all arenas, or all finite arenas. Our main result is that this property is decidable in single exponential time for a given prefix independent $\omega$-regular winning condition. We also exhibit a big class of winning conditions (XPS) which has this property.

Key words: automata, infinite games, omega-regular languages, positional strategies, winning condtions
\end{abstract}

\section{Introduction}

The theory of infinite games is relevant for computer science because of its potential application to verification of interactive systems. In this approach, the system and environment are modeled as players in an infinite game played on a graph (called arena) whose vertices represent possible system states. The players (conventionally called Eve and Adam) decide which edge (state transition, or move) to choose; each edge has a specific color. The desired system's behavior is expressed as a winning condition of the game - the winner depends on the sequence of colors which appear during an infinite play. If a winning strategy exists in this game, the system which implements it will behave as expected. Positional strategies, also called memoryless - ones that depend only on the current position, not on the history of play - are of special interest here, because of their good algorithmic properties which can lead to an efficient implementation.

Infinite games are strongly linked to automata theory. An accepting run of an alternating automaton (on a given tree) can be presented as a winning strategy in a certain game between two players. Parity games are related to automata on infinite structures with parity acceptance condition. For example, positional determinacy of parity games ([Mos91], [EJ91], [McN93]) is used in modern proofs of Rabin's complementation theorem for finite automata on infinite trees with Büchi or parity acceptance condition. See [GTW02] for more links between infinite games, automata, and logic.

An interesting question is, what properties of the winning condition are sufficient for the winning condition to be positionally determined, i.e. guarantee

\footnotetext{
* Supported by KBN grant N206 002 32/0472 and the EC RTN GAMES.
} 
positional winning strategies independently on the arena on which the game is played. Note that although the parity condition gives such strategies for both players, often we need it only for one player (say, Eve). This is true both for interactive systems (we need a simple strategy for ourselves only, we do not care about the environment) and for automata theoretic applications mentioned above (like Rabin's complementation theorem). We call winning conditions with such property half-positional, to make it clear that we require a positional strategy for only one of the players.

Recently some interesting characterizations of positional winning conditions have been found ([CN06], [GZ04], [GZ05], [GW06]; see also [Gra04] for a survey of results on positional determinacy). Our work attempts to obtain similar characterizations and find interesting properties (e.g. closure and decidability properties) of half-positional winning conditions. In this paper we concentrate on winning conditions which are $\omega$-regular.

We show that finite half-positional determinacy of such winning conditions is decidable in singly exponential time. This is done by showing that if a winning condition is not half-positional then there exists a simple arena witnessing this. Then it is possible to check all of such simple arenas. We also show some constructions which lead to half-positional $\omega$-regular winning conditions, such as extended positional/suspendable conditions, concave conditions, and monotonic automata.

This work is a continuation of [Kop06]. Also see the draft [Kop07], which is a superset of both this paper and [Kop06] and contains full proofs which had to be omitted from here due to space limitations.

\section{Preliminaries}

We consider perfect information antagonistic infinite games played by two players, called conventionally Adam and Eve. Let $C$ be a set of colors (possibly infinite).

An arena over $C$ is a tuple $G=\left(\operatorname{Pos}_{A}, \operatorname{Pos}_{E}\right.$, Mov $)$, where:

- Elements of Pos $=\operatorname{Pos}_{E} \cup \operatorname{Pos}_{A}$ are called positions; $\operatorname{Pos}_{A}$ and $\operatorname{Pos}_{E}$ are disjoint sets of Adam's positions and Eve's positions, respectively.

- Elements of Mov $\subseteq$ Pos $\times \operatorname{Pos} \times(C \cup\{\epsilon\})$ are called moves; $\left(v_{1}, v_{2}, c\right)$ is a move from $v_{1}$ to $v_{2}$ colored by $c$. We denote source $\left(v_{1}, v_{2}, c\right)=v_{1}$, $\operatorname{target}\left(v_{1}, v_{2}, c\right)=v_{2}, \operatorname{rank}\left(v_{1}, v_{2}, c\right)=c$.

- $\epsilon$ denotes an empty word, i.e. a colorless move. There are no infinite paths of such colorless moves.

A game is a pair $(G, W)$, where $\mathrm{G}$ is an arena, and $\mathrm{W}$ is a winning condition. A winning condition $W$ over $C$ is a subset of $C^{\omega}$ which is prefix independent, i.e., $u \in W \Longleftrightarrow c u \in W$ for each $c \in C, u \in C^{\omega}$. We name specific winning conditions $W A, W B, \ldots$ For example, the parity condition of rank $n$ is the winning condition over $C=\{0,1, \ldots, n\}$ defined with

$$
W P_{n}=\left\{w \in C^{\omega}: \limsup _{i \rightarrow \infty} w_{i} \text { is even }\right\} .
$$


The game $(G, W)$ carries on in the following way. The play starts in some position $v_{1}$. The owner of $v_{1}$ (e.g. Eve if $v_{1} \in \operatorname{Pos}_{E}$ ) chooses one of the moves leaving $v_{1}$, say $\left(v_{1}, v_{2}, c_{1}\right)$. If the player cannot choose because there are no moves leaving $v_{1}$, he or she loses. The next move is chosen by the owner of $v_{2}$; denote it by $\left(v_{2}, v_{3}, c_{2}\right)$. And so on: in the $n$-th move the owner of $v_{n}$ chooses a move $\left(v_{n}, v_{n+1}, c_{n}\right)$. If $c_{1} c_{2} c_{3} \ldots \in W$, Eve wins the infinite play; otherwise Adam wins.

A play in the arena $G$ is any sequence of moves $\pi$ such that $\operatorname{source}\left(\pi_{n+1}\right)=$ $\operatorname{target}\left(\pi_{n}\right)$. By $\operatorname{source}(\pi)$ and $\operatorname{target}(\pi)$ we denote the initial and final position of the play, respectively. The play can be finite $\left(\pi \in \operatorname{Pos} \cup \mathrm{Mov}^{+}\right.$, where by $\pi \in \operatorname{Pos}$ we represent the play which has just started in the position $\pi$ ) or infinite $\left(\pi \in \mathrm{Mov}^{\omega}\right.$; infinite plays have no target).

A strategy for player $X$ (i.e. $X \in\{$ Eve, Adam $\}$ ) is a partial function $s: \operatorname{Pos} \cup \mathrm{Mov}^{+} \rightarrow$ Mov. Intuitively, $s(\pi)$ for $\pi$ endining in $\operatorname{Pos}_{X}$ says what $X$ should do next. We say that a play $\pi$ is consistent with strategy $s$ for $X$ if for each prefix $\pi^{\prime}$ of $\pi$ such that target $\left(\pi^{\prime}\right) \in \operatorname{Pos}_{X}$ the next move is given by $s\left(\pi^{\prime}\right)$.

A strategy $s$ is winning (for $X$ ) from the position $v$ if $s(\pi)$ is defined for each finite play $\pi$ starting in $v$, consistent with $s$, and ending in $\operatorname{Pos}_{X}$, and each infinite play starting in $v$ consistent with $s$ is winning for $X$.

A strategy $s$ is positional if it depends only on $\operatorname{target}(\pi)$, i.e., for each finite play $\pi$ we have $s(\pi)=s(\operatorname{target}(\pi))$.

A game is determined if for each starting position one of the players has a winning strategy. This player may depend on the starting position in the given play. Thus if the game is determined, the set Pos can be split into two sets $\mathrm{Win}^{E}$ and $\mathrm{Win}^{A}$ and there exist strategies $s_{E}$ and $s_{A}$ such that each play $\pi$ with $\operatorname{source}(\pi) \in \mathrm{Win}^{X}$ and consistent with $s_{X}$ is winning for $X$. All games with a Borel winning condition are determined [Mar75], but there exist (exotic) games which are not determined. A winning condition $W$ is determined if for each arena $G$ the game $(G, W)$ is determined.

We are interested in games and winning conditions for which one or both of the players have positional winning strategies. A determinacy type is given by three parameters: admissible strategies for Eve (positional or arbitrary), admissible strategies for Adam (positional or arbitrary), and admissible arenas (finite or infinite). We say that a winning condition $W$ is $(\alpha, \beta, \gamma)$-determined if for every $\gamma$-arena $G$ the game $(G, W)$ is $(\alpha, \beta)$-determined, i.e. for every starting position either Eve has a winning $\alpha$-strategy, or Adam has a winning $\beta$-strategy. Clearly, there are 8 determinacy types in total. For short, we call (positional, positional, infinite)-determined winning conditions positionally determined or just positional, (positional, arbitrary, infinite)-determined winning conditions half-positional, (arbitrary, positional, infinite)-determined winning conditions co-half-positional. If we restrict ourselves to finite arenas, we add finitely, e.g. (positional, arbitrary, finite)-determined conditions are called finitely halfpositional. For a determinacy type $D=(\alpha, \beta, \gamma), D$-arenas mean $\gamma$-arenas, and $D$-strategies mean $\alpha$-strategies (if they are strategies for Eve) or $\beta$-strategies (for Adam). 
It can be easily proven that a determined game $(G, W)$ is half-positionally determined (i.e. (positional, arbitrary)-determined) iff Eve has a single positional strategy which is winning from each starting position $v \in \operatorname{Win}^{E}(G, W)$.

Note that if a game $(G, W)$ is $(\alpha, \beta)$-determined, then its dual game obtained by using the complement winning condition and switching the roles of players is $(\beta, \alpha)$-determined. Thus, $W$ is $(\alpha, \beta, \gamma)$-determined iff its complement is $(\beta, \alpha, \gamma)$ determined.

We also need following definitions from automata theory.

Definition 1. A deterministic finite automaton on infinite words with parity acceptance condition is a tuple $A=\left(Q, q_{I}, \delta\right.$, rank $)$, where $Q$ is a finite set of states, $q_{I} \in Q$ the initial state, rank $Q \rightarrow\{0, \ldots, d\}$, and $\delta: Q \times C \rightarrow Q$. We extend the definition of $\delta$ to $\delta: Q \times C^{*} \rightarrow Q$ by $\delta(q, \epsilon)=q, \delta(q, w u)=$ $\delta(\delta(q, w), u)$ for $w \in C^{*}, u \in C$. For $w \in C^{\omega}$, let $q_{0}(w)=q_{I}$ and $q_{n+1}(w)=$ $\delta\left(q_{n}, w_{n+1}\right)=\delta\left(q_{I}, w_{0} \ldots w_{n+1}\right)$. We say that the word $w \in C^{\omega}$ is accepted by $A$ iff $\lim \sup _{n \rightarrow \infty} \operatorname{rank}\left(q_{n}(w)\right)$ is even. The set of all words accepted by $A$ is called language accepted by $A$ and denoted $L_{A}$. We say that a language $L \subseteq C^{\omega}$ is $\omega$-regular if it is accepted by some automaton.

\section{$3 \quad$ Types of arenas}

In the games defined above the moves are colored, and it is allowed to have moves without colors. In the literature, several types of arenas are studied.

- $\epsilon$-arenas (C), like the ones described above.

- Move-colored arenas (B). In this setting each move needs to be assigned a color. Moves labelled with $\epsilon$ are not allowed.

- Position-colored arenas (A). In this setting, colors are assigned to positions instead of colors. Instead of Mov $\subseteq \operatorname{Pos} \times \operatorname{Pos} \times C$ we have Mov $\subseteq \operatorname{Pos} \times \operatorname{Pos}$ and a function rank : Pos $\rightarrow C$. Again, each positions needs to be assigned a color. The winner of a play in such games is defined similarly.

If we take a position-colored arena and color each move $m$ with the color $\operatorname{rank}(\operatorname{source}(m))$, we obtain an equivalent move-colored arena. Therefore positioncolored arenas are a subclass of move-colored arenas. Obviously, move-colored arenas are also a subclass of $\epsilon$-colored arenas. When speaking about a determinacy type where we restrict arena to position-colored or move-colored arenas, or we want to emphasize that we allow $\epsilon$-arenas, we add the letter A, B or C (e.g. A-half-positional conditions when we restrict to position-colored arenas).

Hence C-half-positional conditions are a subclass of B-half-positional conditions, and B-half-positional conditions are a subclass of A-half-positional conditions. In the first case the inclusion is proper: there is no way to transform a move-colored arena into a position-colored one such that nothing changes with respect to positional strategies (we can split a position into several new positions according to colors of moves which come into them, but then we obtain new positional strategies which were not positional previously). We know examples of 
winning conditions which are A-positional but not B-positional. One of them is $C^{*}(01)^{*}$; for position-colored arenas we know from our current position to which color we should move next, but not for edge-colored arenas. Another example is min-parity [GW06]. B-positional determinacy has been characterized in [CN06]; this result can be easily generalized to $\epsilon$-arenas. $\epsilon$-arenas have been studied in [Zie98].

In the second case the question whether the inclusion is proper remains open. Note that when we allow $\epsilon$ labels there is no difference whether we label positions or moves: we can replace each move $v_{1} \rightarrow v_{2}$ colored with $c$ in an $\epsilon$-arena with $v_{1} \rightarrow v \rightarrow v_{2}$, color $v$ with $c$, and leave all the original positions colorless.

In this paper we concentrate on $\epsilon$-arenas since we think that this class gives the least restriction on arenas. As shown by the example above, $C^{*}(01)^{*}$, positional strategies for move-colored games are ,,more memoryless” than for position-colored games since they do not even remember the last color used, although winning conditions for position-colored games (like min-parity) may also be interesting. As we will see, it is natural to allow having colorless moves (or equivalently positions).

\section{Simplifying the witness arena}

To show that finite half-positional determinacy of winning conditions which are prefix independent $\omega$-regular languages is decidable, we will first need to show that if $W$ is not finitely half-positional, then it is witnessed by a simple arena.

Theorem 1. Let $W$ be a winning condition accepted by a deterministic finite automaton with parity acceptance condition $A=\left(Q, q_{I}, \delta\right.$, rank $\left.: Q \rightarrow\{0 \ldots d\}\right)$ (see Definition 1). If $W$ is not finitely half-positional then there is a witness arena (i.e. such that Eve has a winning strategy, but no positional winning strategy) where there is only one Eve's position, and only two moves from this position. (There is no restriction on Adam's moves and positions.)

Proof. Let $G$ be any finite witness arena. First, we will show how to reduce the number of Eve's positions to one. Then, we will show how to remove unnecessary moves.

Let $G^{0}=\left(\operatorname{Pos}_{A} \times Q, \operatorname{Pos}_{E} \times Q, \operatorname{Mov}^{0}\right)$ and $G^{1}=\left(\operatorname{Pos}_{A} \times Q, \operatorname{Pos}_{E} \times Q, \operatorname{Mov}^{1}\right)$ where for each move $\left(v_{1}, v_{2}, c\right)$ in $\mathrm{G}$ and each state $q$ we have corresponding moves $\left(\left(v_{1}, q\right),\left(v_{2}, \delta(q, c), c\right)\right)$ in $\operatorname{Mov}^{0}$ and $\left(\left(v_{1}, q\right),\left(v_{2}, \delta(q, c), \operatorname{rank}(q)\right)\right)$ in $\operatorname{Mov}^{1}$. One can easily see that the three games $(G, W),\left(G^{0}, W\right)$ and $\left(G^{1}, W P_{d}\right)$ are equivalent: each play in one of them can be interpreted as a play in each another, and the winner does not change for infinite plays. The games $G^{0}$ and $G$ are equivalent because in $G^{0}$ we just replace each position with a set, and $G^{0}$ and $G^{1}$ are equivalent because A's acceptance parity condition in $\left(G^{0}, W\right)$ uses the same ranks as the parity condition in $\left(G^{1}, W P_{d}\right)$.

Since Eve has a winning strategy in $(G, W)$, she also has a winning strategy in $\left(G^{1}, W P_{d}\right)$. This game is positionally determined, so she also has a positional strategy here. She can use it in $\left(G^{0}, W\right)$ too. 
Let $s$ be Eve's positional winning strategy in $G^{0}$. Let

$$
N(s)=\left\{v: \exists q_{1} \exists q_{2} \pi_{1}\left(\operatorname{target}\left(s\left(v, q_{1}\right)\right)\right) \neq \pi_{1}\left(\operatorname{target}\left(s\left(v, q_{2}\right)\right)\right)\right\},
$$

i.e. the set of positions where $s$ is not positional as a strategy in G. Since the arena is finite, we can assume without loss of generality that there is no positional winning strategy $s^{\prime}$ in $G^{0}$ such that $N\left(s^{\prime}\right) \subsetneq N(s)$.

Obviously, the set $N(s)$ is non-empty. Let $v_{0} \in N(s)$. We construct a new arena $G^{2}$ from $G^{0}$ in two steps. First, merge $\left\{v_{0}\right\} \times Q$ into a single position $v_{0}$. Eve can transform $s$ into a winning strategy $s_{1}$ in this new game - the only difference is that in $v_{0}$ she needs to remember in what state $q$ she is currently, and move according to $s\left(v_{0}, q\right)$. Then, remove all Eve's moves which are not used by $s$ from all Eve's positions except $v_{0}$, and transfer these positions to Adam. Eve still wins, because $s_{1}$ remains a winning strategy after this operation. Thus, we obtained an arena $G^{2}$ with only one Eve's position $v_{0}$ where she has a winning strategy from $v_{0}$.

Eve has no positional strategy in $G^{2}$. Otherwise this strategy could be simulated without changing the winner (in the natural way) by a strategy $s_{1}$ in $G$ which is positional in all positions except $N(s)-\left\{v_{0}\right\}$. This means that there is a positional strategy $s_{2}$ in $G^{0}$ for which $N\left(s_{2}\right) \subseteq N(s)-\left\{v_{0}\right\}$. (We obtain $s_{2}$ by removing from $G$ moves which are not used by $s_{1}\left(s_{1}\right.$ remains a winning strategy) and repeating the construction of $G^{0}$ on the result arena (obtaining a positional strategy on the new arena), and bringing the removed moves back (so our strategy is on $G^{0}$ ). This contradicts our assumption that $N(s)$ is minimal.

Hence, we found a witness arena where $\left|P o s_{E}\right|=1$. To see that we can assume that Eve has at most $|Q|$ moves here, it is enough to see that Eve's finite memory strategy cannot use more than $|Q|$ different moves from this position, hence we can remove the ones which she does not use.

Now, suppose that $G$ is a witness arena with only one Eve's position. We will construct a new arena with only two possible moves for Eve. The construction goes as follows:

- We start with $G^{3}=G^{0}$. Let $s$ be Eve's winning strategy in $G^{3}$.

- For each of Eve's $|Q|$ positions, we remove all moves except the one which is used by $s$.

- (*) Let $v_{1}$ and $v_{2}$ be two Eve's positions in $G^{3}$.

- We merge Eve's positions $v_{1}$ and $v_{2}$ into one, $v_{0}$.

- Eve still has a winning strategy everywhere in this new game (by reasoning similar to one we used for $G^{2}$ ). We check if Eve has a positional winning strategy.

- If yes, we remove the move which is not used in $v_{0}$, and go back to $\left(^{*}\right)$. (Two distinct Eve's positions in $G^{3}$ must still exist — if we were able to merge all Eve's positions into one, it would mean that $G^{3}$ was positionally determined.)

- Otherwise we transfer all Eve's positions other than $v_{0}$ to Adam. There was only one move from each of these positions, hence $G^{3}$ still is a witness arena. 
- In $G^{3}$ we have now only one Eve's position $\left(v_{0}\right)$ and only two Eve's moves

- one inherited from $v_{1}$ and one inherited from $v_{2}$.

\section{Decidability}

Theorem 2. Let $W$ be a (prefix independent) $\omega$-regular winning condition recognized by a DFA with parity acceptance condition $A=\left(Q, q_{I}, \delta\right.$, rank $: Q \rightarrow$ $\{0 \ldots d\})$ with $n$ states. Then finite half-positional determinacy of $W$ is decidable in time $O\left(n^{O\left(n^{2}\right)}\right)$.

Proof. It is enough to check all possible witness arenas which agree with the hypothesis of Theorem 1. Such arena consists of (the only) Eve's position E from which she can move to $A_{1}$ by move $m_{1}$ or to $A_{2}$ by move $m_{2}$. (In general it is possible that $A_{1}$ or $A_{2}$ is equal to $E$. However, this is a simple case and we will concentrate on the one where $A_{1}$ and $A_{2}$ are Adam's positions.) Adam has a choice of word $w$ by which he will return to $E$ from $A_{i}$. (In general it is possible that Adam can choose to never return to $E$. However, if such infinite path would be winning for Eve he would not choose it, and if it would be winning for Adam Eve would never hope to win by choosing to move to $A_{i}$.) Let $L_{i}$ be the set of all possible Adam's return words from $A_{i}$ to $E$.

Let $T(w): Q \rightarrow\{0, \ldots, d\} \times Q$ be the function defined as follows: $T(w)(q)=$ $\left(r, q^{\prime}\right)$ iff $\delta(q, w)=q^{\prime}$ and the greatest rank visited during these transitions is $r$. The function $T(w)$ contains all the information about $w \in L_{i}$ which is important for our game: if $T\left(w_{1}\right)=T\left(w_{2}\right)$ then it does not matter whether Adam chooses to return by $w_{1}$ or $w_{2}$ (the winner does not change). Thus, instead of Adam choosing a word w from $L_{i}$, we can assume that Adam chooses a function $t$ from $T\left(L_{i}\right) \subseteq T\left(C^{*}\right) \subseteq P\left((Q \times Q)^{\{0, \ldots, d\}}\right)$.

For non-empty $R \subseteq\{0, \ldots, d\}$, let best ${ }^{\mathrm{A}}(\mathrm{R})$ be the priority which is the best for Adam, i.e. the greatest odd element of $R$, or the smallest even one if there are no odd priorities in $R$. We also put best ${ }^{\mathrm{A}}(\emptyset)=\perp$.

For $T \subseteq P\left((Q \times Q)^{\{0, \ldots, d\}}\right)$, let

$$
U(T)=\operatorname{best}^{\mathrm{A}}\left(\left\{\mathrm{d}: \exists \mathrm{t} \in \mathrm{T} \mathrm{t}\left(\mathrm{q}_{1}\right)=\left(\mathrm{d}, \mathrm{q}_{2}\right)\right\}\right) .
$$

Again, the function $U_{i}=U\left(T\left(L_{i}\right)\right): Q \times Q \rightarrow\{\perp, 0, \ldots, d\}$ contains all the information about $L_{i}$ which is important for our game - if Adam can go from $q_{1}$ to $q_{2}$ by one of two words $w_{1}$ and $w_{2}$ having the highest priorities $d_{1}$ or $d_{2}$, respectively, he will never want to choose the one which is worse to him.

Our algorithm will check all possible functions $U_{i}$. For this, we need to know whether a particular function $U: Q \times Q \rightarrow\{\perp, 0, \ldots, d\}$ is of form $U\left(T\left(L_{i}\right)\right)$ for some $L_{i}$. This can be done in the following way. We start with $V(q, q)=\perp$. Generate all elements of $T\left(L_{i}\right)$. This can be done by doing a search (e.g. breadth first search) on the graph whose vertices are $T(w)$ and edges are $T(w) \rightarrow T(w c)$ $(T(w c)$ obviously depends only on $T(w))$. For each of these elements, we check if it does not give Adam a better option than $U$ is supposed to give - i.e. for 
some $q_{1}$ we have $T(w c)\left(q_{1}\right)=\left(q_{2}, d\right)$ and $d=\operatorname{best}^{\mathrm{A}}\left(\mathrm{d}, \mathrm{U}\left(\mathrm{q}_{1}, \mathrm{q}_{2}\right)\right)$. If it does not, we add $T(w)$ to our set $T$ and update $V$ : for each $q_{1}, T(w c)\left(q_{1}\right)=\left(q_{2}, d\right)$, we set $V\left(q_{1}, q_{2}\right):=$ best $^{\mathrm{A}}\left(\mathrm{d}, \mathrm{V}\left(\mathrm{q}_{1}, \mathrm{q}_{2}\right)\right)$. If after checking all elements of $T\left(L_{i}\right)$ we get $V=U$, then $U=U(T)$. Otherwise, there is no $L$ such that $U=U(T(L))$.

The general algorithm is as follows:

- Generate all possible functions $U$ of form $U(T(L))$.

- For each possible function $U_{1}$ describing Adam's possible moves after Eve's move $m_{1}$ such that Eve cannot win by always moving with $m_{1}$ :

- For each $U_{2}$ (likewise):

- Check if Eve can win by using a non-positional strategy. (This is done easily by constructing an equivalent parity game which has $3|Q|$ vertices: $\left.\left\{E, A_{1}, A_{2}\right\} \times Q.\right)$ If yes, then we found a witness arena.

Time complexity of the first step is $O\left(d^{O\left(|Q|^{2}\right)}(d|Q|)^{|Q|}|C|\right)$ (for each of $\left.d^{O\left(|Q|^{2}\right.}\right)$ functions, we have to do a BFS on a graph of size $\left.(d|Q|)^{|Q|}\right)$. The parity game in the fourth step can be solved with one of the known algorithm for solving parity games, e.g. with the classical one in time $O\left(O(|Q|)^{d / 2}\right)$. This is done $O\left(d^{O\left(|Q|^{2}\right)}\right)$ times. Thus, the whole algorithm runs in time $\left.O\left(d^{O\left(|Q|^{2}\right.}\right)|Q|^{|Q|}|C|\right)$.

In the proof above the witness arena we find is an $\epsilon$-arena: we did not assign any colors to moves $m_{1}$ and $m_{2}$. If we want to check whether the given condition is A-half-positional or B-half-positional, similar constructions work. For B-halfpositional determinacy, we need to not only choose the sets $U_{1}$ and $U_{2}$, but also assign specific colors $c_{1}$ and $c_{2}$ to both moves $m_{1}$ and $m_{2}$ in the algorithm above. For A-half-positional determinacy, we need to assign specific colors for targets of these two moves, and also a color for Eve's position E.

\section{Examples of $\omega$-regular half-positional winning conditions}

In this section we give some examples of $\omega$-regular half-positional winning conditions. First, we show a class of half-positional winning conditions which generalizes the well known Rabin conditions (see [Kla92,Gra04]) and positional/ suspendable conditions ([Kop06]), and also has some nice closure properties. Note that most of these examples are not restricted to finite arenas. The only exception are the concave conditions, which are only finitely half-positional.

Definition 2. For $S \subseteq C, W B_{S}$ is the set of infinite words where elements of $S$ occur infinitely often, i.e. $\left(C^{*} S\right)^{\omega}$. Winning conditions of this form are called Büchi conditions. Complements of Büchi conditons, $W B_{S}^{\prime}=C^{*}(C-S)^{\omega}$ are called co-Büchi conditions.

Theorem 3 ([Kop06]). Let $D$ be a determinacy type. Let $W \subseteq C^{\omega}$ be a winning condition, and $S \subseteq C$. If $W$ is D-determined, then so is $W \cup W B_{S}$. 
Note that, by duality, Thm 3 implies that if $W$ is $D$-determined, then so is $W \cap W B_{S}^{\prime}$. This yields an easy proof of positional determinacy of parity conditions. It is enough to start with an empty winning condition (which is positionally determined) and apply Thm 3 and its dual $n$ times.

Definition 3 ([Kop06]). A suspendable winning strategy for player $X$ is a pair $(s, \Sigma)$, where $s: \operatorname{Pos} \cup \mathrm{Mov}^{+} \rightarrow \operatorname{Mov}$ is a strategy, and $\Sigma \subseteq \mathrm{Mov}^{*}$, such that:

- $s$ is defined for every finite play $\pi$ such that $\operatorname{target}(\pi) \in \operatorname{Pos}_{X}$.

- every infinite play $\pi$ that is consistent with $s$ from some point $t^{1}$ has a prefix longer than $t$ which is in $\Sigma$;

- Every infinite play $\pi$ that has infinitely many prefixes in $\Sigma$ is winning for $X$.

We say that $X$ has a suspendable winning strategy in $\operatorname{Win}_{X}$ when he has a suspendable winning strategy in the arena $\left(\operatorname{Pos}_{A} \cap \operatorname{Win}_{X}, \operatorname{Pos}_{E} \cap \operatorname{Win}_{X}, \operatorname{Mov} \cap\right.$ $\left.\operatorname{Win}_{X} \times \operatorname{Win}_{X} \times C\right)$.

$A$ winning condition $W$ is positional/suspendable if for each arena $G$ in the game $(G, W)$ Eve has a positional winning strategy in $\mathrm{Win}_{E}$ and Adam has a suspendable winning strategy in $\mathrm{Win}_{A}$.

Intuitively, if at some moment $X$ decides to play consistently with $s$, the play will eventually reach $\Sigma ; \Sigma$ is the set of moments when $X$ can temporarily suspend using the strategy $s$ and return to it later without a risk of ruining his or her victory.

A suspendable winning strategy is a winning strategy, because the conditions above imply that each play which is always consistent with $s$ has infinitely many prefixes in $\Sigma$, and thus is winning for $X$.

The parity condition $W P_{2}$ is positional, but not positional/suspendable, because a suspendable strategy cannot be winning for Adam - it is possible that the play enters state 2 infinitely many times while it is suspended. However, we will now extend the class of positional/suspendable conditions to include also parity (and Rabin) conditions.

Definition 4. The class of extended positional/suspendable (XPS) conditions over $C$ is the smallest set of winning conditions that contains all Büchi and positional/ suspendable conditions, is closed under intersection with coBüchi conditions, and is closed under finite union.

Theorem 4. All XPS conditions are half-positional.

The proof is a modification and generalization of proof of half-positional determinacy of Rabin conditions from [Gra04].

Proof. We use the following lemma:

${ }^{1}$ That is, for each prefix $u$ of $\pi$ which is longer than $t$ and such that $\operatorname{target}(u) \in \operatorname{Pos}_{X}$, the next move is given by $s(u)$. 
Lemma 1 ([Kop06]). Let $D$ be a determinacy type. Let $W \subseteq C^{\omega}$ be a winning condition. Suppose that, for each non-empty $D$-arena $G$ over $C$, there exists a non-empty subset $M \subseteq G$ such that in game $(G, W)$ one of the players has a $D$-strategy winning from $M$. Then $W$ is D-determined. W.

Let $W$ be an XPS condition. The proof is by induction over construction of

We know that Büchi conditions and positional/suspendable conditions are half-positional.

If $W$ is a finite union of simpler XPS conditions, and one of them is a Büchi condition $W B_{S}$, then $W=W^{\prime} \cup W B_{S}$. Then $W^{\prime}$ is half-positional since it is a simpler XPS condition, and from Theorem 3 we get that $W$ is also half-positional.

Otherwise, $W=W^{\prime} \cup \bigcup_{k=1}^{n}\left(W_{k} \cap W B_{S_{k}}^{\prime}\right)$, where $W^{\prime}$ is a positional/ suspendable condition, $W_{k}$ is a simpler XPS condition, and $W B_{S_{k}}^{\prime}$ is a co-Büchi condition. (It is also possible that there is no $W^{\prime}$, but it is enough to consider this case since it is more general. A union of several positional/suspendable conditions is also positional/suspendable [Kop06].) To apply Lemma 1 we need to show that either Eve has a positional winning strategy from some position in the arena, or Adam has a winning strategy everywhere.

For $m=1, \ldots, n$ let $W^{(m)}=W^{\prime} \cup W_{m} \cup \bigcup_{k \neq m}\left(W_{k} \cap W B_{S_{k}}^{\prime}\right)$. We know that $W^{(m)}$ is half-positional since it is a simpler XPS condition.

Let $G$ be an arena. Let $H_{m}$ be the greatest subgraph of $G$ which has no moves colored with any of the colors from $S_{m}$, Adam's positions where he can make a move not in $H_{m}$, and moves which lead to positions which are not in $H_{m}$ (i.e. it is the subgraph where Adam is unable to force doing a move colored with a color from $S_{m}$ ). If Eve has a positional winning strategy from some starting position $v$ in $\left(H_{m}, W^{(m)}\right)$, then she can use the same strategy in $(G, W)$ and win (Eve has more options in G hence she can use the same strategy, and this strategy forces moves colored with $S_{m}$ to never appear).

Assume that Eve has no positional strategy for any starting position and $m$. Then Adam has a following winning strategy in $(G, W)$ :

- Adam uses his suspendable strategy $(s, \Sigma)$ for the game $\left(G, W^{\prime}\right)$, until the play reaches $\Sigma$.

- For $m=1, \ldots, n$ :

- Let $v$ be the current position.

- If $v \in H_{m}$ then Adam uses his winning strategy $s_{m}^{\prime}$ in $\left(H_{m}, W^{(m)}\right)$. (Adam forgets what has happened so far in order to use $s_{m}^{\prime}$.) If Eve never makes a move which does not belong to $H_{m}$ then Adam wins. Otherwise, he stops using $s_{m}^{\prime}$ in some position $v$.

- If $v \notin H_{m}$ then Adam performs a sequence of moves which finally lead to a move colored with $S_{m}$. (He does not need to do that if Eve made a move which is not in $H_{m}$, since it is already colored with $S_{m}$.)

- Repeat.

If finally the game remains in some $H_{m}$, then Adam wins since he is using a winning strategy in $\left(H_{m}, W^{(m)}\right)$. Otherwise, Adam wins $W^{\prime}$ and all the co-Büchi 
conditions $W B_{S_{k}}^{\prime}$ for $k=1, \ldots, n$, hence he also wins $W \subseteq W^{\prime} \cup \bigcup_{k=1}^{n} W B_{S_{k}}^{\prime}$.

Among positional/suspendable (and thus XPS) conditions we have monotonic conditions and geometrical conditions, introduced in [Kop06]. For convenience, we have included the definition of monotonic conditions since they are $\omega$-regular. Monotonic conditions are closed under finite union and include co-Büchi conditions. Another example of a monotonic condition is the complement of the set of words containing $a^{n}$ (alternatively $b a^{n}$ or $a^{n} b$ ) infinitely many times.

Definition 5. A monotonic automaton $A=(n, \delta)$ over an alphabet $C$ is a deterministic finite automaton where:

- the set of states is $Q=\{0, \ldots, n\}$;

- the initial state is 0 , and the accepting state is $n$;

- the transition function $\delta: Q \times C \rightarrow Q$ is monotonic in the first component, i.e., $q \leq q^{\prime}$ implies $\delta(q, c) \leq \delta\left(q^{\prime}, c\right)$.

The language accepted by $A\left(L_{A}\right)$ is the set of words $w \in C^{*}$ such that $\delta(0, w)=n$. A monotonic condition is a winning condition of form $W M_{A}=$ $C^{\omega}-L_{A}^{\omega}$ for some monotonic automaton $A$.

Another interesting class of finitely half-positional winning conditions are the concave conditions, also introduced in [Kop06]. This class is closed under union and includes parity conditions, and $\omega$-regular concave conditions have nice algorithmic properties. Note that concave conditions do not need to be infinitely half-positional (although counterexamples known to us are not $\omega$-regular).

Definition 6. A word $w \in \Sigma^{*} \cup \Sigma^{\omega}$ is a (proper) combination of words $w_{1}$ and $w_{2}$, iff for some sequence of words $\left(u_{n}\right), u_{n} \in \Sigma^{*}, w=\prod_{k \in \mathbb{N}} u_{k}=$ $u_{0} u_{1} u_{2} u_{3} u_{4} u_{5} u_{6} u_{7} u_{8} \ldots, w_{1}=\prod_{k \in \mathbb{N}} u_{2 k+1}=u_{1} u_{3} u_{5} u_{7} \ldots, w_{2}=\prod_{k \in \mathbb{N}} u_{2 k}=$ $u_{0} u_{2} u_{4} u_{6} \ldots$. A winning condition $W$ is convex if as a subset of $C^{\omega}$ it is closed under combinations, and concave if its complement is convex.

Proposition 6.1 ([Kop06]) Suppose that a winning condition $W$ is given by a deterministic parity automaton on infinite words using s states and d ranks. Then there exists a polynomial algorithm determining whether $W$ is concave (or convex). If $W$ is concave and $G$ is an arena with $n$ positions, then the winning sets and Eve's positional strategy can be found in time $O\left(n(n s)^{d / 2} \log s\right)$.

\section{Conclusion and future work}

The main result of this paper is decidability of finite half-positional determinacy of $\omega$-regular conditions (Theorem 2). We also have introduced a large class of half-positionally determined conditions (XPS). This is a generalization of Rabin conditions which includes positional/suspendable ones, such as monotonic conditions introduced in [Kop06]. 
There are still many questions open. Is our algorithm optimal? Showing lower complexity bounds by reducing other known hard problems (e.g. solving parity games) seems difficult in this case. We would have to construct a relevant winning condition (do not forget about prefix independence!), but not the arena - our winning condition is required to have the desired properties for all arenas. It is still possible that simpler characterizations of $\omega$-regular half-positional winning conditions exist.

We have only shown that finite half-positional determinacy is decidable. What about infinite arenas? We conjecture that the classes of finitely halfpositional and half-positional conditions coincide for $\omega$-regular languages, since all examples of finitely half-positional conditions which are not half-positional known to us are not $\omega$-regular. However, a proof is required.

There was also an interesting question raised in section 3. In this paper we assume that not all moves need to be colored. Does this assumption in fact reduce the class of half-positional conditions? The algorithm given in Theorem 2 is more natural when we allow colorless moves, which motivates our opinion that C-half-positional determinacy is a natural notion.

XPS is a very big class, since it generalizes both Rabin conditions and positional/suspendable conditions (including monotonic conditions). This class is closed under finite union; however, we would also like to know whether the whole class of (finitely) half-positional conditions is closed under finite and countable union. In [Kop06] we have shown some more cases where union is also halfpositional, and also that it is not closed under uncountable union.

\section{References}

[CN06] T. Colcombet, D. Niwiński, On the positional determinacy of edge-labeled games. Theor. Comput. Sci. 352 (2006), pages 190-196

[EJ91] E. A. Emerson and C. S. Jutla, Tree automata, mu-calculus and determinacy. Proceedings 32th Annual IEEE Symp. on Foundations of Comput. Sci., pages 368377. IEEE Computer Society Press, 1991.

[Gra04] E. Grädel, Positional Determinacy of Infinite Games. In STACS 2004, LNCS 2996, Springer 2004, pages 4-18.

[GTW02] E. Grädel, W. Thomas, and T. Wilke, eds., Automata, Logics, and Infinite Games. LNCS 2500, Springer-Verlag, 2002.

[GW06] E. Grädel, I. Walukiewicz, Positional determinacy of games with infinitely many priorities. Logical Methods in Computer Science, Vol. 2 (4:6) 2006, pages $1-22$.

[GZ04] H. Gimbert, W. Zielonka, When can you play positionally? Proc. of MFCS '04, LNCS 3153, Springer 2004, pages 686-697.

[GZ05] H. Gimbert, W. Zielonka, Games Where You Can Play Optimally Without Any Memory. CONCUR 2005, LNCS 3653, Springer 2005, pages 428-442.

[Kla92] N. Klarlund, Progress measures, immediate determinacy, and a subset construction for tree automata. Proc. 7th IEEE Symp. on Logic in Computer Science, 1992.

[Kop06] E. Kopczyński, Half-Positional Determinacy of Infinite Games. M. Bugliesi et al. (Eds.), ICALP 2006, Part II, LNCS 4052, Springer 2006, pages 336-347. 
[Kop07] E. Kopczyński, Half-positional determinacy of infinite games. Draft. http://www.mimuw.edu.pl/ erykk/papers/hpwc.ps

[Mar75] D. A. Martin, Borel determinacy. Ann. Math., 102:363-371, 1975.

[McN93] R. McNaughton, Infinite games played on finite graphs. Annals of Pure and Applied Logic, 65:149-184, 1993.

[Mos91] A. W. Mostowski, Games with forbidden positions. Technical Report 78, Uniwersytet Gdański, Instytut Matematyki, 1991.

[Zie98] W. Zielonka, Infinite Games on Finitely Coloured Graphs with Applications to Automata on Infinite Trees. Theor. Comp. Sci. 200(1-2): 135-183 (1998) 